\title{
Do Personality Traits Moderate Relations Between Psychologically Controlling Parenting and Problem Behavior in Adolescents?
}

\author{
Elien Mabbe,' Bart Soenens,' Maarten Vansteenkiste,' and \\ Karla Van Leeuwen ${ }^{2}$ \\ 'Ghent University \\ ${ }^{2}$ KULeuven
}

\begin{abstract}
This research examined whether and how adolescents' personality traits moderate associations between psychologically controlling parenting and problem behaviors. On the basis of self-determination theory, we also examined the mediating role of psychological need frustration in the effects of psychologically controlling parenting. A cross-sectional study in two samples ( $N=423$ and 292; $M_{\text {age }}=12.43$ and I 5.74 years) was conducted. While in Sample I both mothers and adolescents provided reports of parenting and problem behavior, Sample 2 relied on adolescent-reported parenting and motherreported problem behavior. Psychologically controlling parenting was related to internalizing and externalizing problems in both samples. Little systematic evidence was obtained for the moderating role of personality, with the exception of a moderating effect of Agreeableness. In both samples, psychological control was unrelated to externalizing problems among adolescents high on Agreeableness. Analyses of Sample 2 showed that associations between psychological control and problem behavior were mediated by psychological need frustration. Adolescent personality plays a modest role as a moderator of associations between psychologically controlling parenting and problem behavior. Frustration of adolescents' basic and universal psychological needs can account for the undermining effects of psychologically controlling parenting. Directions for future research are discussed.
\end{abstract}

Psychologically controlling parenting (i.e., parenting characteristic of parents who use intrusive and sometimes subtle tactics such as guilt induction and love withdrawal; Barber, 1996) is predictive of maladjustment in children (Barber \& Xia, 2013; Soenens \& Vansteenkiste, 2010). One intriguing yet largely unaddressed question is whether psychological control is related to maladjustment in all children or whether these associations occur only in children with particular personality characteristics. While previous research has addressed the moderating role of personality in effects of harsh and more explicit forms of parental control, the moderating role of personality in effects of psychologically controlling parenting has not been addressed yet. In addition, we examined the role of the frustration of basic and universal psychological needs as a mediator explaining effects of psychologically controlling parenting, as articulated within self-determination theory (Deci \& Ryan, 2000). These research questions were examined in two samples of early and middle adolescents.

\section{Psychologically Controlling Parenting}

Psychologically controlling parenting involves the use of intrusive and often insidious parental tactics to pressure the child and to manipulate the parent-child bond, including guilt induction, love withdrawal, and shaming (Barber, 1996). Barber (1996) argued that psychological control primarily yields an emotional cost for the child, as manifested in internalizing problems. Psychological control might elicit at least some compliance with parental requests because children do not want to disappoint their parents (Assor, Roth, \& Deci, 2004). Therefore, children of psychologically controlling parents may not necessarily display externalizing problems. Initial work by Barber, Olsen, and Shagle (1994) showed that psychological control was related

We are grateful to Barbara De Clercq and to Peter Prinzie for having read and commented upon an earlier version of this paper.

Correspondence concerning this article should be addressed to Elien Mabbe, Department of Developmental, Personality, and Social Psychology, H. Dunantlaan 2, Belgium. Email: elien.mabbe@ugent.be. 
uniquely to internalizing but not to externalizing problems. This original pattern of findings was replicated, with studies showing a systematic and unique association with internalizing problems and a relatively more inconsistent association with externalizing problems (Barber \& Xia, 2013; Soenens \& Vansteenkiste, 2010).

The association between psychological control and problem behaviors has been found to be quite robust, with effects being documented across different age groups (e.g., Aunola \& Nurmi, 2005) and across diverse cultures (e.g., Ahmad, Vansteenkiste, \& Soenens, 2013). Although age and culture do not appear to systematically moderate effects of psychologically controlling parenting, the moderating role of personality and the mechanisms that can account for these robust effects have been examined less.

\section{A Self-Determination Theory Perspective on Psychologically Controlling Parenting}

To account for the effects of psychologically controlling parenting across age and culture, it has been argued on the basis of self-determination theory (SDT; Deci \& Ryan, 2000) that this parenting style thwarts universal psychological needs in children (Soenens \& Vansteenkiste, 2010). These needs are defined as "innate psychological nutriments that are essential for ongoing psychological growth, integrity, and well-being" (Deci \& Ryan, 2000, p. 229). The need for autonomy refers to experiences of volition and psychological freedom. When frustrated, the need for autonomy manifests in feelings of pressure and coercion. The need for competence refers to the experience of mastery over the environment. When frustrated, it manifests in feelings of inadequacy. The need for relatedness refers to the experience of reciprocal care and love in the relation to significant others, including parents. When frustrated, this need manifests in feelings of loneliness and isolation. Testifying to the universal importance of these needs, research conducted in different age groups (e.g., Veronneau, Koestner, \& Abela, 2005) and across different cultures (e.g., Chen et al., 2015) has confirmed that need satisfaction is related to well-being and that need frustration is related to maladjustment (e.g., Bartholomew, Ntoumanis, Ryan, \& Thogersen-Ntoumani, 2011; Vansteenkiste \& Ryan, 2013).

Consistent with the presumed explanatory role of these needs, a few recent studies in different cultures showed that psychologically controlling parenting is related to child maladjustment through its association with low need satisfaction (or even need frustration; Ahmad et al., 2013; Costa, Soenens, Gugliandolo, Cuzzocrea, \& Larcan, 2015). While findings from these studies further confirm that psychologically controlling parenting is universally detrimental, the role of children's personality in effects of this parenting dimension has not yet been addressed. An examination of the potentially moderating role of personality is critical because if effects of psychologically controlling parenting strongly depend on personality, the claim that psychological control appeals to universal psychological needs would be disconfirmed.

\section{Parenting $\times$ Personality Interactions}

The notion that child characteristics moderate the effects of parenting in predicting developmental outcomes is rooted in several conceptual models (Kiff, Lengua, \& Zalewski, 2011). To illustrate, the goodness-of-fit model (Thomas, Chess, \& Birch, 1968) suggests that adaptation and development are fostered when parental characteristics match or are congruent with individuals' characteristics. Against the background of the general notion of goodness of fit, more specific models have been developed. Diathesis-stress models (Monroe \& Simons, 1991), for instance, maintain that children with a susceptible personality may be more vulnerable to the effects of adverse parenting. More recently, the differential susceptibility hypothesis highlights the more general idea of children's differential responsiveness to parenting (Belsky, 1997). Children with a susceptible personality would not only suffer more from adverse parenting but would also benefit more from an absence of negative parenting or from positive parenting.

Most research on the moderating role of personality in effects of parenting has relied on the Five-Factor Model of personality (i.e., Emotional Stability, Extraversion, Openness to Experience, Agreeableness, and Conscientiousness; Caspi \& Shiner, 2006) and has included measures of harsh and explicit forms of controlling parenting (e.g., coercive discipline; Prinzie et al., 2003; Van Leeuwen, Mervielde, Braet, \& Bosmans, 2004). To the best of our knowledge, to date no studies have examined interactions with psychologically controlling parenting. Although the number of significant interactions found in earlier research was rather limited in light of the number of interactions tested, some interactions emerged consistently. Specifically, Agreeableness and Conscientiousness have been found to buffer effects of harsh parental control on externalizing problems (De Clercq, Van Leeuwen, De Fruyt, Van Hiel, \& Mervielde, 2008; de Haan, Prinzie, \& Dekovic, 2010; Prinzie et al., 2003; Van Leeuwen et al., 2004; Van Leeuwen, Mervielde, De Clercq, \& De Fruyt, 2007) and internalizing problems (Van Leeuwen et al., 2007) in a broad age range ( $7-15$ years).

\section{Toward a Nuanced Perspective on the Moderating Role of Personality}

Although at first sight the hypotheses derived from SDT (according to which psychologically controlling parenting is universally maladaptive) may seem inconsistent with the literature on Parenting $\times$ Personality interactions (according to which psychologically controlling parenting would be detrimental only for adolescents with certain personality features), two important nuances need to be made. First, consideration needs to be given to the nature of the interaction. Most previously documented interactions are ordinal in nature, meaning that the strength but 
not the direction of the relationship between controlling parenting and problem behavior is influenced by the moderator. While personality affects the degree to which children suffer from controlling parenting, it is not the case that some children benefit from controlling parenting. The SDT perspective would be disconfirmed only when psychological control would be beneficial for some adolescents or when it would be systematically unrelated to any type of problem behavior in some adolescents.

A second nuance concerns the idea that personality may primarily shape the manifestation of the costs associated with psychological control. Although SDT predicts that psychological control is universally harmful, it is less clear about the way maladjustment is expressed. This manifestation may depend on personality differences, with psychological control yielding primarily externalizing and internalizing problems among, respectively, adolescents scoring high on undercontrolled traits (e.g., low Conscientiousness) and adolescents scoring high on overcontrolled traits (e.g., high Neuroticism/low Emotional Stability). Consistent with this reasoning, Zarra-Nezhad et al. (2014) recently showed in a sample of elementary school children that psychologically controlling parenting was related positively to internalizing problems and negatively related to externalizing problems only among children high on social withdrawal.

\section{The Present Research}

The present study examined, first, the role of Big Five personality traits in the relation between psychologically controlling parenting and children's problem behaviors and, second, the explanatory role of psychological need frustration in this association. These two issues were pursued in a sample of early adolescents (Sample 1) and a sample of middle adolescents (Sample 2) and their mothers. Both samples make use of different informants, with Sample 1 involving both mother and adolescent reports of both parenting and problem behavior and with Sample 2 relying on adolescent reports of psychological control and mother reports of problem behavior. Because interaction effects can be quite sample-specific and unstable, it was deemed important to replicate the moderating effects of personality across two independent samples so as to have more confidence in the interaction findings obtained.

We focused on mothers because they continue to represent key socialization figures in early to middle adolescents' lives (Maccoby \& Martin, 1983). Further, we sampled adolescents because adolescence is known to be characterized by increasing independence and individuation from parents, who may interfere with this development through a controlling approach. Both research on the main effects of psychologically controlling parenting (Soenens \& Vansteenkiste, 2010) and research on the main effects of the Big Five personality traits (e.g., Meeus, Van de Schoot, Klimstra, \& Branje, 2011) have shown that associations with problem behaviors are typically similar throughout adolescence. Yet, as individual differences in personality become more stable and crystallized throughout adolescence (e.g., Klimstra, Hale, Raaijmakers, Branje, \& Meeus, 2009), it could be argued that personality plays a stronger moderating role with increasing age. In contrast, on the basis of SDT, we expected that psychologically controlling parenting would yield fairly similar developmental correlates across both samples and across individuals with different scores on the Big Five dimensions. If any interactions would emerge, we expected them to be ordinal in nature and/or to specify the manifestation of maladjustment associated with psychological control (in terms of internalizing or externalizing problems). Also on the basis of SDT, we hypothesized that need frustration would mediate associations between psychologically controlling parenting and problem behaviors and that this mediation model would be largely invariant across individual differences in personality.

In examining this set of hypotheses, we addressed the role of gender. Although mean-level gender differences have been reported with regard to both psychologically controlling parenting (e.g., with boys receiving somewhat more psychological control than girls; Barber, Bean, \& Erickson, 2002) and problem behaviors (e.g., with boys scoring higher on externalizing problems and girls scoring higher on internalizing problems; Leadbeater, Kuperminc, Blatt, \& Hertzog, 1999), associations between psychologically controlling parenting and problem behaviors are typically invariant across gender (Soenens \& Vansteenkiste, 2010).

\section{METHOD}

\section{Participants and Procedure}

Sample 1 consisted of 423 Belgian, Dutch-speaking adolescents $\left(M_{\mathrm{age}}=12.43\right.$ years, $S D=1.13$, range $=10-16 ; 53 \%$ female $)$ and their mothers $\left(M_{\mathrm{age}}=41.14, S D=3.93\right.$, range $\left.=31-55\right)$. The adolescents were recruited via elementary and secondary schools by three research assistants. Their mothers received a letter describing the goal of the study and requesting them to fill out a form if they would not allow their son or daughter to participate in the study (passive informed consent). The mothers were also invited to participate in the study themselves. If they agreed, they completed an enclosed questionnaire, which they returned in a sealed and coded envelope. Adolescents who agreed to participate in the study completed a questionnaire in class during a free hour, under the supervision of a research student. The overall response rate was $83 \%$. Regarding educational level, $40.4 \%$ of the mothers completed secondary school, $46.7 \%$ had a bachelor's degree, and $11.8 \%$ attained a master's degree.

As for the adolescents, $47.5 \%$ were in the last grades of primary school (5th or 6th grade) and $52.5 \%$ were in the first grades of secondary school (7th or 8th grade). Concerning family status, $89.4 \%$ were part of two-parent families.

Sample 2, a unique sample with no overlap with Sample 1, consisted of 292 adolescents $\left(M_{\text {age }}=15.74\right.$ years, $S D=1.21$, range $=12-19 ; 56 \%$ female) and their mothers, recruited by undergraduate psychology students during home visits. Of all 
participating adolescents, seven indicated that they did not have the Belgian nationality. Still, these participants spoke Dutch and lived in Belgium. Mothers provided active informed consent. Participants were ensured that all information would be treated confidentially. Participation was voluntary, and anonymity was guaranteed. Their mothers had a mean age of 45.56 years $(S D=$ 4.20), with a range between 29 and 59 years. Regarding educational level, $0.4 \%$ of the mothers completed elementary school, $40.8 \%$ completed secondary school, $42.2 \%$ had a bachelor's degree, and $16.7 \%$ attained a master's degree. Concerning family status, $81.9 \%$ came from two-parent families.

\section{Measures}

Psychologically Controlling Parenting (Samples I and 2). Adolescents (Samples 1 and 2) and mothers (Sample 1) were administered the well-validated and frequently used Psychological Control Scale-Youth Self-Report (PCS-YSR; Barber, 1996). The scale includes eight items (e.g., "My mother is / I am always trying to change how I / my child feel(s) or think(s) about things") that were scored on a 5-point Likert scale ranging from 1 (completely not true) to 5 (completely true). Cronbach's alphas for mother- and adolescent-reported psychological control were, respectively, .58 and .74 in Sample 1 and .76 for adolescent report in Sample 2. In Sample 1, we decided not to create a composite score for psychologically controlling parenting (aggregating across informants) because the correlation between maternal and adolescent reports was relatively low (i.e., $r=.19, p<.01)$. Moreover, we aimed to examine the moderating role of personality for maternal and adolescent reports of psychologically controlling parenting separately. To create latent factors for adolescent-reported and mother-reported psychologically controlling parenting, we created for each construct three parcels that consisted of randomly assigned items (Little, Cunningham, Shahar, \& Widaman, 2002).

Internalizing and Externalizing Problems (Samples I and 2). In both samples, mothers were administered the Child Behavior Checklist (CBCL; Achenbach, 1991). Adolescents in Sample 1 were additionally administered the Youth Self-Report (YSR; Achenbach, 1991). Items were scored on a 3-point Likert scale ranging from 0 (never) to 2 (often). The broadband scale Internalizing Problems $(\alpha=.90$ and .85 for adolescent and mother report, respectively, in Sample 1 and .86 for mother report in Sample 2) consists of three syndrome scales: Anxious/ Depressed (e.g., “... cries a lot”), Withdrawn/Depressed (e.g., “... enjoys little"), and Somatic Complaints (e.g., “... has headaches"). The broadband scale Externalizing Problems ( $\alpha=.85$ and .88 for adolescent and mother report, respectively, in Sample 1 and .90 for mother report in Sample 2) consists of two syndrome scales: Rule Breaking (e.g., “... drinks alcohol") and Aggressive Behavior (e.g., “... destroys others' things”). Similar to previous research (e.g., Achenbach, McConaughy, \& Howell, 1987), the correlation between mother and adolescent reports of internalizing and externalizing problems in Sample 1 was .43 and .40 , respectively. To create latent factors for internalizing and externalizing problems in Sample 1, both constructs were represented by their respective subscales. Because mother and adolescent reports of internalizing and externalizing problems were moderately correlated, we combined maternal and adolescent ratings to obtain a composite multi-informant score for each subscale of internalizing and externalizing problems. To do so, mother and adolescent reports on all subscales were first standardized and then averaged across the adolescent and mother reports. In Sample 2, internalizing and externalizing problems were both represented by their respective subscales.

Personality (Samples I and 2). Adolescents in Samples 1 and 2 completed the Quick Big Five (QBF; Vermulst \& Gerris, 2005). Research has shown that the QBF is a valid measure of adolescents' Big Five personality traits because it correlates with measures of adjustment and problem behavior much like other measures of Big Five personality traits (Dubas, Gerris, Janssens, \& Vermulst, 2002). Previous research has also shown strong correlations between self-rated QBF scores and parental ratings of personality using the Hierarchical Personality Inventory for Children (HiPIC) (Van Leeuwen, De Fruyt, \& Mervielde, 2004).

The QBF includes 30 adjectives, six items for each of the Big Five personality traits. Examples of items are "careful" (Conscientiousness), "talkative" (Extraversion), "helpful" (Agreeableness), "nervous" (Emotional Stability, reverse scored) and "innovative" (Openness to Experience). Internal consistencies across samples varied between .61 and .90 , with an average of .79. Each item was rated on a 7-point Likert scale ranging from 1 (completely incorrect) to 7 (completely correct). To create latent factors of each Big Five personality trait, they were each represented by three parcels that consisted of randomly assigned items.

Need Frustration (Sample 2 Only). Adolescents in Sample 2 reported on experiences of need frustration specifically in the mother-child relationship ( $\alpha=.85$ ). Recently, Chen et al. (2015) developed and validated the Basic Psychological Need Satisfaction and Need Frustration Scale (BPNSNF) to tap into both need satisfaction and need frustration in general. We slightly adapted the items to the parent-child relation and only retained the items tapping into need frustration, as need frustration has been found to account for the effects of controlling socialization in other life domains (e.g., Vansteenkiste \& Ryan, 2013). Frustration of each of the three needs was measured with three items, each beginning with "When I'm with my mother": autonomy need frustration (e.g., "... I feel forced to do many things I wouldn't choose to do"), competence frustration (e.g., “... I feel insecure about my abilities"), and relatedness frustration (e.g., "... I feel often rejected"). Items were scored on a 5point Likert scale ranging from 1 (completely not true) to 5 (completely true). A latent factor was created with the three subscales as indicators. 
Table I Sample I: Descriptive Statistics and Correlations Between Variables

\begin{tabular}{|c|c|c|c|c|c|c|c|c|c|c|c|c|c|c|c|}
\hline & I & 2 & 3 & 4 & 5 & 6 & 7 & 8 & 9 & 10 & II & 12 & 13 & 14 & 15 \\
\hline 3. Extraversion & $-.17^{* *}$ & -.03 & & & & & & & & & & & & & \\
\hline 4. Conscientiousness & $-.15 * *$ & -.08 & .01 & & & & & & & & & & & & \\
\hline $\begin{array}{l}\text { 7. Openness to } \\
\text { Experience }\end{array}$ & .01 & -.07 & .08 & $.19 * *$ & $.40 * *$ & -.07 & & & & & & & & & \\
\hline 8. Internalizing (A) & $.35^{* *}$ & .10 & $-.4 I^{* *}$ & -.09 & $-.20 * *$ & $-.54 * *$ & -.01 & & & & & & & & \\
\hline 9. Externalizing (A) & $.40 * *$ & .09 & $-.16^{*}$ & $-.30 * *$ & $-.30 * *$ & $-.26^{* *}$ & -.06 & $.56 * *$ & & & & & & & \\
\hline I0. Internalizing (M) & $.19 * *$ & $.32^{* *}$ & $-.17^{* *}$ & -.08 & $-.15 * *$ & $-.29 * *$ & -.09 & $.43^{* *}$ & $.33 * *$ & & & & & & \\
\hline II. Externalizing (M) & $.19 * *$ & $.24^{* *}$ & -.03 & $-.2 I^{* *}$ & $-.15^{* *}$ & $-.18^{* *}$ & .02 & $.21^{* *}$ & $.40 * *$ & $.58 * *$ & & & & & \\
\hline$M$ & 1.90 & 1.68 & 4.93 & 4.32 & 5.56 & 4.08 & 4.72 & .41 & .27 & .22 & .17 & 1.55 & 1.71 & 12.43 & 3.83 \\
\hline$S D$ & .65 & .44 & 1.04 & 1.23 & .82 & 1.16 & .94 & .29 & .20 & .19 & .17 & .50 & 1.84 & 1.13 & 1.17 \\
\hline
\end{tabular}

Note. $N=423$. $(A)=$ adolescent reported; $(M)=$ mother reported.

$* p<.05$ and $*_{p} p<.01$.

\section{RESULTS}

\section{Preliminary Analyses}

Means, standard deviations, and correlations between the study variables are presented in Table 1 (Sample 1) and Table 2 (Sample 2). To determine whether participants' scores on the study variables varied by gender, age, family status, and maternal educational level, a MANOVA was conducted. There was an overall multivariate effect for gender, Sample 1: Wilks's $\lambda=.84$, $F(297)=6.20, p<.001$; Sample 2: Wilks's $\lambda=.78, F(253)=$ 4.07, $p<.001$, and age, Sample 1: Wilks's $\lambda=.92, F(297)=$ 2.87, $p<.01$; Sample 2: Wilks's $\lambda=.84, F(253)=2.65, p<$ .001 . There were no multivariate effects for family status, Sample 1: Wilks's $\lambda=.95, F(297)=1.91, n s ;$ Sample 2: Wilks's $\lambda$ $=.91, F(253)=1.33, n s$, and maternal educational level, Sample 1: Wilks's $\lambda=.98, F(297)=.62, n s ;$ Sample 2: Wilks's $\lambda=$ $.94, F(253)=.88, n s$. Follow-up univariate analyses revealed that girls rated themselves higher on Conscientiousness (Sample $1: M=4.46, S D=1.24$; Sample $2: M=3.85, S D=1.30$ ) than boys (Sample 1: $M=4.13, S D=1.19$; Sample 2: $M=3.51$, $S D=1.18)$, whereas they scored lower on Emotional Stability (Sample 1: $M=3.95, S D=1.21$ ) than boys (Sample 1: $M=$ $4.26, S D=1.07)$. Further, in Sample 1, girls reported fewer externalizing problems $(M=.25, S D=.19)$ than boys $(M=$ $.30, S D=.21)$, but they reported more internalizing problems $(M=.45, S D=.31)$ than boys $(M=.36, S D=.26)$. In Sample 2, girls reported less psychologically controlling parenting $(M=2.23, S D=.67)$ and less autonomy frustration $(M=2.43$,
$S D=.70)$ than boys $(M=2.41, S D=.58 ; M=2.75, S D=$ $.80)$. Finally, older adolescents reported being more conscientious and more open-minded in Sample 1. In Sample 2, older adolescents reported less Extraversion and were rated as scoring higher on internalizing problems by their mothers. Gender and age were included as control variables in the main analyses.

\section{Primary Analyses}

Basic Measurement and Structural Models. Structural equation modeling with MPlus (Muthén \& Muthén, 1998 2012) was performed to examine the hypotheses. To evaluate model fit, the comparative fit index (CFI), the root mean square error of approximation (RMSEA), and the standardized root mean square residual (SRMR) were selected. According to $\mathrm{Hu}$ and Bentler (1999), combined cut-off values close to .95 for CFI and close to .06 for RMSEA and .09 for the SRMR indicate good fit. We controlled for gender and age by allowing paths from both variables to all study variables. Prior to estimating the structural models, in both samples we evaluated the measurement models (including all constructs). In Sample 1, the measurement models including adolescent-reported, $\chi^{2}(202)=$ 500.80; RMSEA $=.06 ; \mathrm{CFI}=.91 ;$ SRMR $=.06 ; \mathrm{TLI}=.89$; factor loadings ranging between .61 and .94 (all $p$ s $<.001$ ), and mother-reported, $\chi^{2}(202)=491.75 ;$ RMSEA $=.06$; CFI $=.91$; SRMR $=.06 ;$ TLI $=.89$; factor loadings ranged between .60 and .96 (all $p \mathrm{~s}<.001$ ), parenting showed adequate fit. In 
Table 2 Sample 2: Descriptive Statistics and Correlations Between Variables

\begin{tabular}{|c|c|c|c|c|c|c|c|c|c|c|c|c|c|}
\hline & 1 & 2 & 3 & 4 & 5 & 6 & 7 & 8 & 9 & 10 & $\mathrm{II}$ & 12 & 13 \\
\hline 2. Need frustration $(A)$ & $.61 * *$ & & & & & & & & & & & & \\
\hline 4. Externalizing problems (M) & $.43 * *$ & $.36 * *$ & $.44 * *$ & & & & & & & & & & \\
\hline 5. Extraversion (A) & -.00 & $-.20 * *$ & $-.29 * *$ & $.12^{*}$ & & & & & & & & & \\
\hline 6. Conscientiousness (A) & $-.13 *$ & $-.14^{*}$ & -.04 & $-.22 * *$ & -.10 & & & & & & & & \\
\hline 9. Openness to Experience (A) & .01 & -.09 & -.02 & .03 & $.12 *$ & .07 & $.27^{* *}$ & $-.12 *$ & & & & & \\
\hline 10. Gender & $.14 *$ & $.14 *$ & .03 & .07 & -.08 & $-.13^{*}$ & $-.12^{*}$ & $.26 * *$ & .04 & & & & \\
\hline II. Family status & .02 & .04 & .04 & $.16 * *$ & -.03 & $-.15^{*}$ & $-.14^{*}$ & -.06 & -.00 & -.10 & & & \\
\hline 12. Adolescent age & .02 & -.06 & $.12^{*}$ & .06 & $-.20 * *$ & -.08 & -.04 & .06 & .06 & .01 & .01 & & \\
\hline 13. Maternal educational level & -.09 & .05 & .00 & -.03 & -.00 & -.03 & .01 & -.04 & .05 & .03 & -.03 & -.03 & \\
\hline M & 2.30 & 2.15 & .26 & .21 & 4.94 & 3.72 & 5.34 & 4.11 & 4.60 & .42 & 1.20 & 15.74 & 3.95 \\
\hline
\end{tabular}

Note. $N=292$. (A) = adolescent reported; $(M)=$ mother reported

$*_{p}<.05$ and $*^{*} p<.01$.

Sample 2, the measurement model also showed adequate fit, $\chi^{2}(263)=581.46 ;$ RMSEA $=.06 ;$ CFI $=.90 ;$ SRMR $=.06$; $\mathrm{TLI}=.87$. Factor loadings ranged between .48 and .89 (all $p \mathrm{~s}<$ $.001)$.

Next, we estimated structural models including associations between psychological control and both internalizing and externalizing problems, with the latter two variables being allowed to correlate. The models showed adequate fit, Sample 1: $\chi^{2}(29)=$ 78.78; $\mathrm{RMSEA}=.07$; $\mathrm{CFI}=.95 ; \mathrm{SRMR}=.04$; TLI $=.93$ for adolescent-reported parenting; $\chi^{2}(29)=84.91$; RMSEA $=.07$; $\mathrm{CFI}=.94 ; \mathrm{SRMR}=.04 ; \mathrm{TLI}=.91$ for mother-reported parenting; Sample 2: $\chi^{2}(29)=88.94$; RMSEA $=.09$; CFI $=.90$; $\mathrm{SRMR}=.06 ; \mathrm{TLI}=.85$. Psychological control was positively associated with both internalizing $(\beta=.43, p<.001$, and $\beta=$ $.31, p<.001$, for adolescent and mother reports, respectively, in Sample $1 ; \beta=.24, p<.05$ in Sample 2$)$ and externalizing $(\beta=$ $.43, p<.001$, and $\beta=.25, p<.01$, for adolescent and mother reports, respectively, in Sample $1 ; \beta=.47, p<.001$ in Sample 2) problems. Multigroup analyses revealed that gender did not moderate associations in the structural models (Sample 1: $\Delta \chi^{2}$ $=4.95, d f=2, p>.05$ for the model with adolescent-reported psychological control and $\Delta \chi^{2}=.08, d f=2, p>.05$ for the model with mother-reported psychological control; Sample 2: $\left.\Delta \chi^{2}=.03, d f=2, p>.05\right)$.

Next, a full mediation model in Sample 2, in which psychological control was related only indirectly to the outcomes through need frustration, $\chi^{2}(58)=154.03$; RMSEA $=.08$; CFI $=.90 ; \mathrm{SRMR}=.07 ; \mathrm{TLI}=.87$, showed that psychologically controlling parenting was related to need frustration $(\beta=.80 p$ $<.001)$, which, in turn, was related to internalizing $(\beta=.31 p$ $<.001)$ and externalizing problems $(\beta=.43, p<.001)$. Adding direct paths from psychological control to problem behaviors in addition to the indirect paths did not improve model fit, $\Delta \chi^{2}(2)$ $=6.77, p>.01$, suggesting that the full mediation model was the best-fitting model. In the full mediation model, psychological control had significant indirect effects (through need frustration) on both internalizing $(\beta=.24, p<.001)$ and externalizing problems $(\beta=.35, p<.001)$. Multigroup analysis revealed that gender did not moderate associations in this structural model $\left(\Delta \chi^{2}=4.89, d f=3, p>.05\right)$.

Parenting $\times$ Personality Interactions. We tested the moderating role of the Big Five traits by entering each of the personality dimensions in separate analyses. Since MPlus provides only limited information about fit for moderation analyses (Muthén \& Muthén, 1998-2012), it has been recommended to first test the main effects of the predictors (i.e., psychologically controlling parenting and personality), without considering potential interactions between the parenting and personality variables (Maslowsky, Jager, \& Hemken, 2015). Given that these models showed adequate fit (RMSEA $=.06-.08$; CFI $=.91-$ $.96 ; \mathrm{SRMR}=.04-.06$ ), we proceeded by adding the interaction terms. Results are shown in Table 3.

Emotional Stability, Extraversion (Samples 1 and 2), and Agreeableness (Sample 1) yielded an independent negative association with internalizing problems, whereas Conscientiousness, Agreeableness (Sample 1), and Emotional Stability (Samples 1 and 2) were significantly negatively related to externalizing problems. Psychologically controlling parenting consistently emerged as a positive predictor of both internalizing and externalizing problems in both samples, even when controlling for the contribution of the Big Five traits. Out of the 20 interactions tested in Sample 1, two were significant for internalizing problems and two were significant for externalizing problems. Three of the four interactions involved mother-reported parenting, and only one involved adolescent-reported parenting. Across the 10 interaction tests in Sample 2, one significant interaction emerged. 
Table 3 Unstandardized Beta Coefficients and Standard Errors of the Main and Interaction Effects of Controlling Parenting and Personality

\begin{tabular}{|c|c|c|c|c|c|c|}
\hline & \multicolumn{4}{|c|}{ Sample I } & \multicolumn{2}{|c|}{ Sample 2} \\
\hline & \multicolumn{2}{|c|}{ Internalizing } & \multicolumn{2}{|c|}{ Externalizing } & \multirow[t]{2}{*}{ Internalizing } & \multirow[t]{2}{*}{ Externalizin } \\
\hline & \multicolumn{4}{|c|}{ Parenting Report } & & \\
\hline & Adolescent & Mother & Adolescent & Mother & Adolescent & Adolescent \\
\hline Psychologically controlling parenting & $.12(.03)^{* *}$ & $.21(.06)^{* *}$ & $.08(.02)^{* *}$ & $.09(.03)^{* *}$ & $.08(.04)^{*}$ & $.15(.04)^{* *}$ \\
\hline Extraversion & $-.12(.02)^{* *}$ & $-.14(.03)^{* *}$ & $-.01(.01)$ & $-.02(.01)$ & $-.07(.02)^{* *}$ & $.01(.01)$ \\
\hline Parenting $\times$ Extraversion & $-.07(.05)$ & $-.23(.09)^{* *}$ & $-.03(.04)$ & $-.10(.05)$ & $-.03(.05)$ & $-.04(.04)$ \\
\hline Psychologically controlling parenting & $.15(.03)^{* *}$ & $.19(.06)^{* *}$ & $.07(.01)^{* *}$ & $.09(.05)^{*}$ & $.09(.04)^{*}$ & $.16(.04)^{* *}$ \\
\hline Conscientiousness & $-.02(.02)$ & $-.03(.02)$ & $-.03(.01)^{* *}$ & $-.03(.01)^{* *}$ & $-.00(.02)$ & $-.03(.02)$ \\
\hline Parenting $\times$ Conscientiousness & $.03(.02)$ & $-.01(.08)$ & $-.01(.01)$ & $-.05(.06)$ & $-.06(.05)$ & $-.06(.04)$ \\
\hline Psychologically controlling parenting & $.14(.03)^{* *}$ & $.17(.05)^{* *}$ & $.07(.02)^{* *}$ & $.08(.02)^{* *}$ & $.08(.04)^{*}$ & $.13(.03)^{* *}$ \\
\hline Agreeableness & $-.08(.03)^{* *}$ & $-.08(.02)^{* *}$ & $-.03(.01)^{* *}$ & $-.04(.01)^{* *}$ & $-.03(.03)$ & $-.03(.02)$ \\
\hline Parenting $\times$ Agreeableness & $-.01(.04)$ & $-.10(.07)$ & $-.06(.02)^{* *}$ & $-.14(.04)^{* *}$ & $-.15(.09)$ & $-.13(.06)^{*}$ \\
\hline Psychologically controlling parenting & $.10(.03)^{* *}$ & $.30(.15)^{*}$ & $.06(.02)^{* *}$ & $.10(.05)^{*}$ & $.09(.04)^{*}$ & $.12(.04)^{* *}$ \\
\hline Emotional Stability & $-.14(.02)^{* *}$ & $-.14(.02)^{* *}$ & $-.03(.01)^{* *}$ & $-.04(.01)^{* *}$ & $-.06(.02)^{* *}$ & $-.02(.01)^{*}$ \\
\hline Parenting $\times$ Emotional Stability & $-.06(.05)$ & $-.24(.12)^{*}$ & $-.03(.02)$ & $-.07(.04)$ & $-.06(.05)$ & $-.05(.03)$ \\
\hline Psychologically controlling parenting & $.15(.03)^{* *}$ & $.20(.06)^{* *}$ & $.08(.02)^{* *}$ & $.08(.02)^{* *}$ & $.10(.04)^{*}$ & $.14(.04)^{* *}$ \\
\hline Openness to Experience & $-.01(.02)$ & $.00(.03)$ & $.00(.01)$ & $.01(.01)$ & $.01(.02)$ & $.01(.01)$ \\
\hline Parenting $\times$ Openness to Experience & $.04(.06)$ & $.08(.14)$ & $.00(.03)$ & $.01(.04)$ & $.03(.05)$ & $.04(.04)$ \\
\hline
\end{tabular}

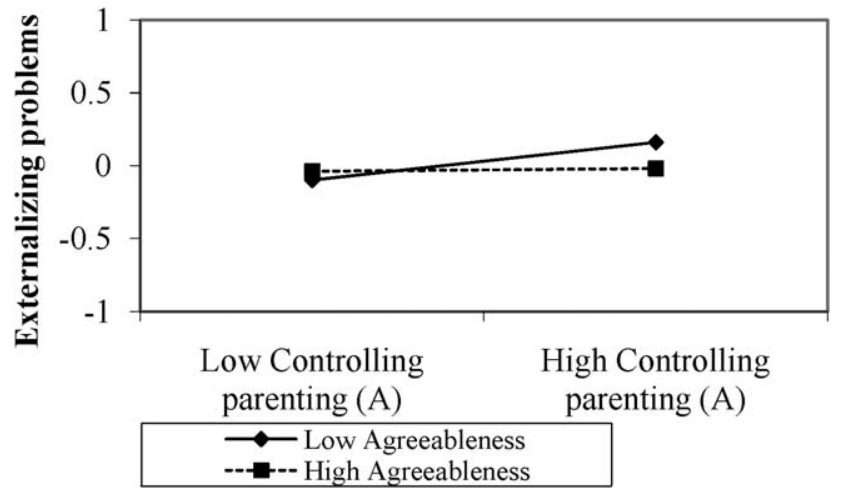

Figure I Sample I: Interaction between adolescent-reported controlling parenting and Agreeableness in the prediction of externalizing problems.

To interpret significant interactions, we inspected associations between psychologically controlling parenting and the outcomes at low (one standard deviation below the mean) and high (one standard deviation above the mean) levels of the moderator through simple slope analyses (Aiken \& West, 1991). Motherreported (Sample 1) and adolescent-reported (Samples 1 and 2) psychologically controlling parenting were found to interact with Agreeableness in the prediction of externalizing problems. These three interactions were very similar. Psychologically controlling parenting was related positively to externalizing problems in low Agreeableness adolescents (Sample 1: $\beta=.22, p<$ $.001 ; \beta=.13, p<.01$; Sample $2: \beta=.26, p<.01$ ), yet it was unrelated to externalizing problems among adolescents high in

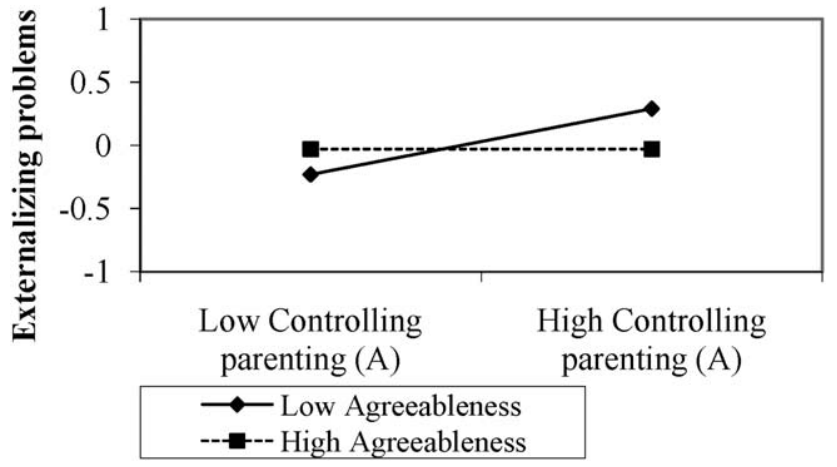

Figure 2 Sample 2: Interaction between adolescent-reported controlling parenting and Agreeableness in the prediction of externalizing problems.

Agreeableness (Sample 1: $\beta=-.06, p>.05 ; \beta=.01, p>.05$; Sample 2: $\beta=.00, p>.05$ ). Figure 1 (Sample 1) and Figure 2 (Sample 2) illustrate these interactions with the case of adolescent-reported psychological control.

Mother-reported psychologically controlling parenting interacted with both Extraversion and Emotional Stability in the prediction of internalizing problems in Sample 1. Given the similarity of these interactions, only the interaction involving Extraversion is displayed in Figure 3. Mother-reported psychological control related positively to internalizing problems for adolescents low in Extraversion $(\beta=.45, p<.001)$ and low in Emotional Stability $(\beta=.54, p<.05)$, yet it was unrelated to internalizing problems for adolescents high in Extraversion 


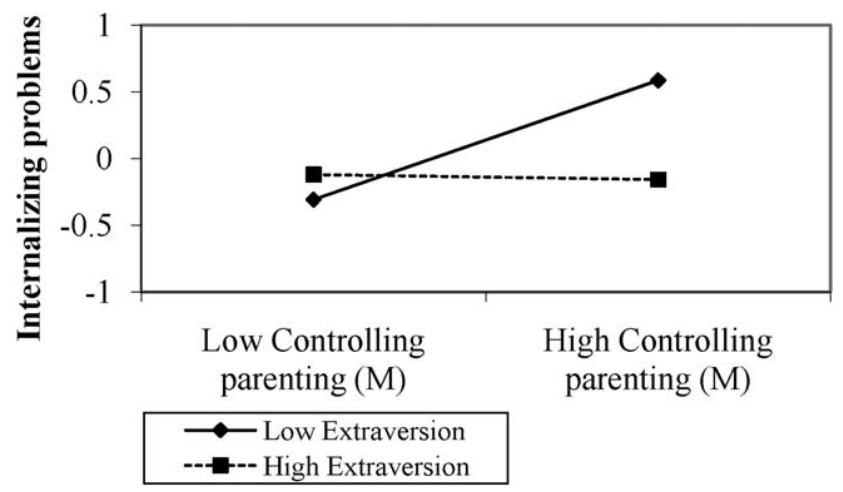

Figure 3 Sample I: Interaction between mother-reported controlling parenting and Extraversion in the prediction of internalizing problems.

$(\beta=-.02, p>.05)$ and high in Emotional Stability $(\beta=.06$, $p>.05)$.

Next, we entered the Big Five dimensions as moderators in the mediation model in Sample 2. None of the five possible interactions between the personality dimensions and psychological control in the prediction of need frustration reached significance. Further, none of the 10 possible interactions between the personality dimensions and need frustration in the prediction of the two types of problem behaviors reached significance. ${ }^{1}$

\section{GENERAL DISCUSSION}

Dozens of studies showed that psychologically controlling parenting hampers children's development (Barber \& Xia, 2013; Soenens \& Vansteenkiste, 2010). However, the question of whether psychological control is related to maladjustment irrespective of children's personality has received only little attention. This study aimed to examine whether the effects of maternal psychological control are limited to adolescents with particular personality traits or whether, instead, its effects generalize across individual differences between adolescents. Further, we examined whether the frustration of the psychological needs for autonomy, competence, and relatedness served as a mechanism explaining why psychologically controlling parenting has universally undermining effects (Soenens \& Vansteenkiste, 2010).

\section{Do the Correlates of Psychological Control Depend on the Adolescents' Personality?}

Consistent with previous research (e.g., Asendorpf, Borkenau, Ostendorf, \& Van Aken, 2001), Agreeableness and Emotional Stability were related negatively to both externalizing and internalizing problems. Conscientiousness was primarily negatively related to externalizing problems, whereas Extraversion showed in particular negative associations with internalizing problems. Also consistent with previous research (e.g., Barber, 1996), psychologically controlling parenting was related to both internalizing and externalizing problems, regardless of whether mothers or children provided ratings of psychologically controlling parenting. Moreover, in both samples, psychological control was predictive of problem behaviors above and beyond the variance explained by the Big Five traits, which underscores the robustness of the maladaptive developmental outcomes associated with parental psychological control.

The most innovative part of the study, however, was the examination of the potential moderating role of adolescents' personality. A number of observations can be made regarding both the nature and the number of interactions obtained. Regarding the nature of the interactions obtained, we found that these interactions were ordinal and not crossover in nature. When personality played a moderating role, it changed the strength (but not the direction) of the effect of psychologically controlling parenting. Overall, findings suggest that the association between psychologically controlling parenting and problems is weakened (sometimes to nonsignificance) for adolescents with certain personality traits. Yet, this does not mean that some individuals benefited from controlling parenting. Adolescents paid at least some price when being exposed to psychologically controlling parenting, either in the form of internalizing or externalizing problems.

With regard to the number of interactions, across both samples and the 30 interactions that were tested, only five turned out to be significant (i.e., 17\%). Given this limited number of significant interactions, the moderating role of personality can be considered as modest. One interaction did replicate across both samples and across informants of psychologically controlling parenting. Specifically, adolescents scoring low on Agreeableness were most vulnerable to the adverse effects of parental psychological control. Conversely, psychological control was unrelated to externalizing problems among highly agreeable adolescents, suggesting that Agreeableness serves as a protective factor against the adverse effects of psychologically controlling parenting. It appears that adolescents high on Agreeableness do not act against their environment in response to pressures experienced at home. This finding is strikingly similar to findings obtained with other types of controlling parenting, such as overreactivity and overt harshness (e.g., de Haan et al., 2010; Van Leeuwen, Mervielde, Braet, \& Bosmans, 2004).

The reason why the interaction occurred with Agreeableness and not with the other personality traits may be explained by the fact that Agreeableness is critical for interpersonal functioning. Jensen-Campbell, Gleason, Adams, and Malcolm (2003) described Agreeableness as an "interpersonally oriented personality characteristic" (p. 1061). Further, Rothbart and Bates (1998) suggested that Agreeableness may emerge from effortful control and, as such, is critical for the way people deal with interpersonal stressors. Given that psychological control represents an interpersonal source of frustration and stress, it becomes intelligible why this parenting style interacts with Agreeableness in particular. Indeed, highly agreeable adolescents have been found to perceive less interpersonal conflict and to display more adaptive modes of conflict resolution (e.g., Graziano, JensenCampbell, \& Hair, 1996). In light of these findings, we forward 
two specific yet rather speculative explanations for why Agreeableness buffers the effects of psychologically controlling parenting (see also Soenens, Vansteenkiste, \& Van Petegem, 2015). First, adolescents high on Agreeableness may be less likely to interpret potentially psychologically controlling behavior as intrusive and pressuring. Second, even when the behavior is perceived as intrusive, they may cope with this experience more effectively (e.g., by engaging in negotiation with parents; Skinner, Edge, Altman, \& Sherwood, 2003).

It is important to note, however, that Agreeableness did not moderate associations of psychological control with internalizing problems. Hence, although children high on Agreeableness do not respond to psychologically controlling parenting with externalizing problems, they do display internalizing problems; that is, they experience internal distress. These findings help explain why associations of psychological control with externalizing problems are relatively less unique and consistent than those with internalizing problems (Barber \& Xia, 2013; Soenens $\&$ Vansteenkiste, 2010). Associations with externalizing problems are somewhat more conditional upon child personality and adolescents' Agreeableness in particular.

Apart from this fairly stable interaction, a few other interactions emerged. In Sample 1, mother-reported but not adolescentreported psychological control was unrelated to internalizing problems among adolescents high on Extraversion and Emotional Stability. The lack of moderation in the case of adolescent-perceived psychological control is in line with the notion that adolescent perceptions of parenting ultimately determine their adjustment. Specifically, when adolescents perceive their mother to be psychologically controlling, they invariantly report heightened internalizing distress, irrespective of differences in personality (Soenens et al., 2015).

\section{Understanding the Mechanisms Underlying Psychologically Controlling Parenting}

The observation that adolescents, regardless of their personality traits, seem to pay at least some price for exposure to psychologically controlling parenting is consistent with SDT. From the SDT perspective, the correlates of psychologically controlling parenting should largely generalize across personality because it frustrates adolescents' basic psychological needs, which are presumed to be universally critical (Deci \& Ryan, 2000). When being raised by a psychologically controlling parent, children would feel pressured to think, act, or behave in particular ways (i.e., autonomy frustration), would feel inadequate because of the parent's critical and negative tone of communication (i.e., competence frustration), and would feel a sense of alienation in the parent-child relationship because of the parent's conditional acceptance (i.e., relatedness frustration; Soenens \& Vansteenkiste, 2010).

Consistent with this reasoning, results from Sample 2 provided evidence for need frustration as a critical mediator through which psychologically controlling parenting relates to maladap- tive outcomes. These results are important because although it has been postulated that psychological control exerts its effects through processes of need frustration (Soenens \& Vansteenkiste, 2010), only a few studies directly addressed this hypothesis (see, e.g., Ahmad et al., 2013, for an exception). The current study is unique in that it focused specifically on experiences of need frustration rather than on an absence of need satisfaction. Recent SDT-based studies suggest that social conditions that actively thwart children's needs do more than just provide insufficient levels of support for children's needs (Bartholomew et al., 2011; Haerens, Aelterman, Vansteenkiste, \& Soenens, 2015). Psychologically controlling parents do not simply afford little autonomy; they actively block children in their pursuits and direct them toward their own standards, thereby eliciting feelings of compulsion. Also, it has been hypothesized and found that experiences of need thwarting and need frustration are more strongly predictive of psychopathology than an absence of need support and need satisfaction (e.g., Bartholomew et al., 2011; Vansteenkiste \& Ryan, 2013). Given that psychological control can be considered a parenting strategy that actively thwarts children's needs, it seemed appropriate to focus in the present study on need frustration as a mediator rather than on low need satisfaction.

Importantly, the presumed explanatory role of need frustration was found to be operative irrespective of specific personality dimensions. As such, the findings are consistent with SDT's assumption that the needs are universally important and that their active thwarting relates to maladjustment for everyone (Deci \& Ryan, 2000).

\section{LIMITATIONS AND DIRECTIONS FOR FUTURE RESEARCH}

The current study is limited by its use of a cross-sectional design. Although personality may not buffer effects of psychologically controlling parenting strongly in the short run, it might do so in the longer run. Also, longitudinal studies increasingly show that the associations between psychologically controlling parenting and maladjustment are reciprocal (e.g., Soenens, Luyckx, Vansteenkiste, Duriez, \& Goossens, 2008). As such, personality may play a role in both directions of effects. For instance, adolescents' personality may moderate effects of problem behavior on parental psychological control (e.g., such that parents respond more strongly with psychological control to problem behavior when the adolescent simultaneously displays more maladaptive personality features). At the same time, parents' own personality may play a role in parental reactions to problem behaviors (e.g., such that parents scoring high on adaptive personality features are less inclined to respond to adolescent problem behavior with psychologically controlling behavior).

Another limitation is the exclusive focus on maternal use of psychological control. Most research suggests that the dynamics of maternal and paternal psychologically controlling parenting are fairly similar (Barber \& Xia, 2013; Soenens \& 
Vansteenkiste, 2010). Yet it remains to be tested whether the moderating role of personality also operates similarly across parental gender. A third limitation is that the two samples could not be directly and formally compared (e.g., through multigroup analysis) because somewhat different assessment procedures were used in both samples. As such, the moderating role of age needs further attention in future research.

A fourth limitation is that we relied on a relatively brief and broad measure of Big Five personality. Although broad, higherorder dimensions of personality may not moderate effects of psychologically controlling parenting systematically, it might be the case that more specific, lower-level dimensions of personality do. Fifth, for some scales in Sample 1, the reliability was low. This problem was dealt with by modeling all constructs as latent variables (thereby controlling for error variance). Still, the findings with these scales in Sample 1 need to be interpreted with some caution. More generally, parent reports of psychological control may be affected by social desirability, which future research could control for.

Sixth, the lack of adolescent (in addition to mother) reports of problems in Sample 2 is a limitation, particularly with regard to internalizing problems, as adolescents themselves may be the most important source of information. To gain insight in the role of the informant, we performed an additional set of analyses on the Sample 1 data, thereby separating mother and child reports of problem behavior. These analyses showed that both the main effects and interaction effects obtained with the total (multi-informant) scores for internalizing and externalizing problems were also obtained with the separate scores for problem behaviors. Such findings suggest that the restriction to mother reports of problem behavior in Sample 2 probably did not affect the findings. Still, future research may systematically include adolescent reports of problem behavior.

Although the sample size of our study was substantial, future research may rely on even larger samples. Indeed, statistical interactions are notoriously difficult to find for simple statistical reasons related to effect and sample size. At the same time, one may wonder whether interactions that show up only in very large samples are meaningful and sufficiently large in terms of effect size. Although we obtained few systematic moderating effects of personality, it is premature to conclude that the role of personality in the dynamics of psychologically controlling parenting can be dismissed. In this regard, it is important to note that we measured psychologically controlling parenting through self-reports and mostly even through children's self-reports. Although children are at increased risk to display problem behavior as soon as they perceive their parents to be psychologically controlling, one may wonder how children come to construct perceptions of psychologically controlling parenting and whether personality plays a role in this process of perceiving parental behavior (Soenens et al., 2015). One and the same parental statement (e.g., "I am quite disappointed by your most recent exam results") may be interpreted quite differently by children with different personality traits. Perhaps, then, the moderating role of personality does not need to be situated in between children's perceptions of parents and the developmental consequences but in between parents' actual behavior and the child's perception of parental behavior. Future research can address this hypothesis.

\section{CONCLUSION}

The current study showed that maternal psychological control has robust associations with problem behaviors in adolescents, even when controlling for individual differences in personality. Some evidence was obtained for a moderating role of personality, with Agreeableness in particular buffering effects of maternal psychological control on externalizing (but not internalizing) problems. Overall, personality did not have a strong or systematic moderating role. Although further research (using longitudinal designs and separating actual parental behavior from how it is perceived) is needed, the current results are in line with the notion that, in one way or another, psychologically controlling parenting is detrimental to adolescents, irrespective of their personality traits.

\section{Declaration of Conflicting Interests}

The authors declared no potential conflicts of interest with respect to the research, authorship, and/or publication of this article.

\section{Funding}

The authors received no financial support for the research, authorship, and/or publication of this article.

\section{Note}

1. Analyses were also conducted excluding multivariate outliers. Multivariate outliers were identified with the Mahalanobis distance measure (Kim, 2000; Penny, 1996). Six multivariate outliers in Sample 1 and eight multivariate outliers in Sample 2 were removed. In Sample 1, all main effects of psychologically controlling parenting and the personality variables remained essentially the same. With respect to the interaction effects, the interaction between motherreported psychological control and Emotional Stability in the prediction of internalizing problems was no longer significant. The other interactions that reached significance in the analyses with the full sample also reached significance in the analyses without the outliers. There was an additional significant interaction between motherreported psychologically controlling parenting and Extraversion in the prediction of externalizing problems. In Sample 2, all main effects of psychologically controlling parenting and personality variables also remained the same. The one significant interaction that 
was found in the analyses with the full sample was no longer significant when the outliers were deleted.

\section{References}

Achenbach, T. M. (1991). Manual for the Child Behavior Checklist/ 4-18 and 1991 profiles. Burlington: University of Vermont, Department of Psychiatry.

Achenbach, T. M., McConaughy, S. H., \& Howell, C. T. (1987). Child adolescent behavioral and emotional problems: Implications of cross-informant correlations for situational specificity. Psychological Bulletin, 101, 213-232.

Ahmad, I., Vansteenkiste, M., \& Soenens, B. (2013). The relations of Arab Jordanian adolescents' perceived maternal parenting to teacher-rated adjustment and problems: The intervening role of perceived need satisfaction. Developmental Psychology, 49, 177183.

Aiken, L. S., \& West, S. G. (1991). Multiple regression: Testing and interpreting interactions. Newbury Park, CA: Sage.

Asendorpf, J. B., Borkenau, P., Ostendorf, F., \& Van Aken, M. A. G. (2001). Carving personality description at its joints: Confirmation of three replicable personality prototypes for both children and adults. European Journal of Personality, 15, 169-198.

Assor, A., Roth, G., \& Deci, E. L. (2004). The emotional costs of parents' conditional regard: A self-determination theory analysis. Journal of Personality, 72, 47-88.

Aunola, K., \& Nurmi, J. E. (2005). The role of parenting styles in children's problem behavior. Child Development, 76, 1144-1159.

Barber, B. K. (1996). Parental psychological control: Revisiting a neglected construct. Child Development, 67, 3296-3319.

Barber, B. K., Bean, R. L., \& Erickson, L. D. (2002). Expanding the study and understanding of psychological control. In B. K. Barber (Ed.), Intrusive parenting: How psychological control affects children and adolescents (pp. 263-289). Washington, DC: American Psychological Association.

Barber, B. K., Olsen, J. E., \& Shagle, S. C. (1994). Associations between parental psychological and behavioral control and youth internalized and externalized behaviors. Child Development, $\mathbf{6 5}$, $1120-1136$.

Barber, B. K., \& Xia, M. (2013). The centrality of control to parenting and its effects. In R. E. Larzelere, A. S. Morris, \& A. W. Harrist (Eds.), Authoritative parenting: Synthesizing nurturance and discipline for optimal child development (pp. 61-87). Washington, DC: American Psychological Association Press.

Bartholomew, K. J., Ntoumanis, N., Ryan, R. M., \& ThogersenNtoumani, C. (2011). Psychological need thwarting in the sport context: Assessing the darker side of athletic experience. Journal of Sport \& Exercise Psychology, 33, 75-102.

Belsky, J. (1997). Variation in susceptibility to environmental influence: An evolutionary argument. Psychological Inquiry, 8, $182-186$.

Caspi, A., \& Shiner, R. L. (2006). Personality development. In N. Eisenberg (Ed.), Handbook of child psychology: Vol. 3. Social, emotional, and personality development (pp. 300-365). New York: Wiley.
Chen, B., Vansteenkiste, M., Beyers, W., Boone, L., Deci, E. L., Duriez, B., et al. (2015). Basic psychological need satisfaction, need frustration, and need strength across four cultures. Motivation and Emotion, 39, 216-236.

Costa, S., Soenens, B., Gugliandolo, M. C., Cuzzocrea, F., \& Larcan, R. (2015). The mediating role of experiences of need satisfaction in associations between parental psychological control and internalizing problems: A study among Italian college students. Journal of Child and Family Studies, 24, 1106-1116.

Deci, E. L., \& Ryan, R. M. (2000). The "what" and "why" of goal pursuits: Human needs and the self-determination of behavior. Psychological Inquiry, 11, 227-268.

De Clercq, B., Van Leeuwen, K., De Fruyt, F., Van Hiel, A., \& Mervielde, I. (2008). Maladaptive personality traits and psychopathology in childhood and adolescence: The moderating effect of parenting. Journal of Personality, 76, 357-383.

de Haan, A. D., Prinzie, P., \& Dekovic, M. (2010). How and why children change in aggression and delinquency from childhood to adolescence: Moderation of overreactive parenting by child personality. Journal of Child Psychology and Psychiatry, 51, $725-733$.

Dubas, J. S., Gerris, J. R. M., Janssens, J., \& Vermulst, A. A. (2002). Personality types of adolescents: Concurrent correlates, antecedents, and type $\times$ parenting interactions. Journal of Adolescence, 25, 79-92.

Graziano, W. G., Jensen-Campbell, L. A., \& Hair, E. C. (1996). Perceiving interpersonal conflict and reacting to it: The case for agreeableness. Journal of Personality and Social Psychology, 70, $820-835$.

Haerens, L., Aelterman, N., Vansteenkiste, M., \& Soenens, B. (2015). Do perceived autonomy-supportive and controlling teaching relate to physical education students' motivational experiences through unique pathways? Distinguishing between the bright and dark side of motivation. Psychology of Sport and Exercise, 16, 26-36.

Hu, L. T., \& Bentler, P. M. (1999). Cutoff criteria for fit indexes in covariance structure analysis: Conventional criteria versus new alternatives. Structural Equation Modeling: A Multidisciplinary Journal, 6, 1-55.

Jensen-Campbell, L. A., Gleason, K. A., Adams, R., \& Malcolm, K. T. (2003). Interpersonal conflict, agreeableness, and personality development. Journal of Personality, 71, 1059-1086.

Kiff, C. J., Lengua, L. J., \& Zalewski, M. (2011). Nature and nurturing: Parenting in the context of child temperament. Clinical Child and Family Psychology Review, 14, 251-301.

Kim, M. G. (2000). Multivariate outliers and decompositions of Mahalanobis distance. Communications in Statistics: Theory and Methods, 29, 1511-1526.

Klimstra, T. A., Hale, W. W., Raaijmakers, Q. A. W., Branje, S. J. T., \& Meeus, W. H. J. (2009). Maturation of personality in adolescence. Journal of Personality and Social Psychology, 96, 898912.

Leadbeater, B. J., Kuperminc, G. P., Blatt, S. J., \& Hertzog, C. (1999). A multivariate model of gender differences in 
adolescents' internalizing and externalizing problems. Developmental Psychology, 35, 1268-1282.

Little, T. D., Cunningham, W. A., Shahar, G., \& Widaman, K. F. (2002). To parcel or not to parcel: Exploring the question, weighing the merits. Structural Equation Modeling, 9, 151-173.

Maccoby, E. E., \& Martin, J. A. (1983). Socialization in the context of the family: Parent-child interaction. In P. H. Mussen \& E. M. Hetherington, Handbook of child psychology: Vol. 4. Socialization, personality, and social development (4th ed., pp. 1-101). New York: Wiley.

Maslowsky, J., Jager, J., \& Hemken, D. (2015). Estimating and interpreting latent variable interactions: A tutorial for applying the latent moderated structural equations method. International Journal of Behavioral Development, 39, 87-96.

Meeus, W., Van de Schoot, R., Klimstra, T., \& Branje, S. (2011). Personality types in adolescence: Change and stability and links with adjustment and relationships: A five-wave longitudinal study. Developmental Psychology, 47, 1181-1195.

Monroe, S. M., \& Simons, A. D. (1991). Diathesis stress theories in the context of life stress research: Implications for the depressive disorders. Psychological Bulletin, 110, 406-425.

Muthén, L. K., \& Muthén, B. O. (1998-2012). Mplus user's guide (7th ed.). Los Angeles: Muthén \& Muthén.

Penny, K. I. (1996). Appropriate critical values when testing for a single multivariate outlier by using the Mahalanobis distance. Applied Statistics: Journal of the Royal Statistical Society Series C, 45, 73-81.

Prinzie, P., Onghena, P., Hellinckx, W., Grietens, H., Ghesquiere, P., \& Colpin, H. (2003). The additive and interactive effects of parenting and children's personality on externalizing behaviour. European Journal of Personality, 17, 95-117.

Rothbart, M. K., \& Bates, J. (1998). Temperament. In W. Damon (Series Ed.) \& N. Eisenberg (Vol. Ed.), Handbook of child psychology: Vol. 3. Social, emotional, and personality development (5th ed., pp. 105-176). New York: Wiley.

Skinner, E. A., Edge, K., Altman, J., \& Sherwood, H. (2003). Searching for the structure of coping: A review and critique of category systems for classifying ways of coping. Psychological Bulletin, 129, 216-269.

Soenens, B., Luyckx, K., Vansteenkiste, M., Duriez, B., \& Goossens, L. (2008). Clarifying the link between perceived parental psychological control and adolescents' depressive feelings: Reciprocal versus unidirectional models of influence. Merrill-Palmer Quarterly, 54, 411-444.

Soenens, B., \& Vansteenkiste, M. (2010). A theoretical upgrade of the concept of parental psychological control: Proposing new insights on the basis of self-determination theory. Developmental Review, 30, 74-99.

Soenens, B., Vansteenkiste, M., \& Van Petegem, S. (2015). Let us not throw out the baby with the bathwater: Applying the principle of universalism without uniformity to autonomy-supportive and controlling parenting. Child Development Perspectives, 9, 44-49.

Thomas, A., Chess, S., \& Birch, H. (1968). Temperament and behavior disorders in children. New York: New York University Press.

Van Leeuwen, K., De Fruyt, F., \& Mervielde, I. (2004). A longitudinal study of the utility of the resilient, overcontrolled, and undercontrolled personality types as predictors of children's and adolescents' problem behaviour. International Journal of Behavioral Development, 28, 210-220.

Van Leeuwen, K., Mervielde, I., Braet, C., \& Bosmans, G. (2004). Child personality and parental behavior as moderators of problem behavior: Variable- and person-centered approaches. Developmental Psychology, 40, 1028-1046.

Van Leeuwen, K., Mervielde, I., De Clercq, B. J., \& De Fruyt, F. (2007). Extending the spectrum idea: Child personality, parenting and psychopathology. European Journal of Personality, 21, 63-89.

Vansteenkiste, M., \& Ryan, R. M. (2013). On psychological growth and vulnerability: Basic psychological need satisfaction and need frustration as a unifying principle. Journal of Psychotherapy Integration, 23, 263-280.

Vermulst, A. A., \& Gerris, J. R. M. (2005). QBF: Quick Big Five Persoonlijkheidstest, Handleiding [Quick Big Five personality test manual]. Leeuwarden, the Netherlands: LDC.

Veronneau, M. H., Koestner, R. F., \& Abela, J. R. Z. (2005). Intrinsic need satisfaction and well-being in children and adolescents: An application of the self-determination theory. Journal of Social and Clinical Psychology, 24, 280-292.

Zarra-Nezhad, M., Kiuru, N., Aunola, K., Zarra-Nezhad, M., Ahonen, T., Poikkeus, A.-M., et al. (2014). Social withdrawal in children moderates the association between parenting style and the children's own socioemotional development. Journal of Child Psychology and Psychiatry, 55, 1260-1269. 